\title{
Loss of interstitial Cajal-like cells in the gallbladder might contribute to gallstone formation
}

Interstitial Cajal-like cells (ICLCs) present in the gallbladder might have a role in the pathogenesis of gallstones, according to a study published in Neurogastroenterology \& Motility.

The mechanisms behind the formation of gallstones are not clearly understood, but gallbladder dysmotility seems to be a key factor. Given that interstitial cells of Cajal are known to have an important role in regulating gut motility, and the growing evidence that ICLCs (as they are known outside the gastrointestinal tract) are present in the gallbladder, a team of researchers decided to investigate the role of ICLCs in gallstone disease.

"During one of our lab meetings with Professors Andrzej Matyja and Piotr Thor, we wondered whether ICLCs are present in the gallbladder wall of patients with cholelithiasis, and whether their density decreases or remains intact compared with patients without cholelithiasis," explain Krzysztof Gil and Artur Pasternak, two authors of the study. "Having some scientific background and an experienced laboratory ready, we put together a small group of surgeons and morphologists to perform the study."

The researchers used indirect double immunofluorescence to visualize ICLCs in tissue samples from 30 patients with gallstones and 25 patients without gallstones. These cells were observed throughout the gallbladder, although predominantly in the muscularis propria of the corpus. Gil, Pasternak and colleagues also found that the density of ICLCs in the muscularis propria was significantly lower in patients with gallstones than in those without $(P<0.001)$. This reduced density of ICLCs might affect gallbladder motility.

The researchers conclude that their findings need to be confirmed using other methods, but they tentatively suggest

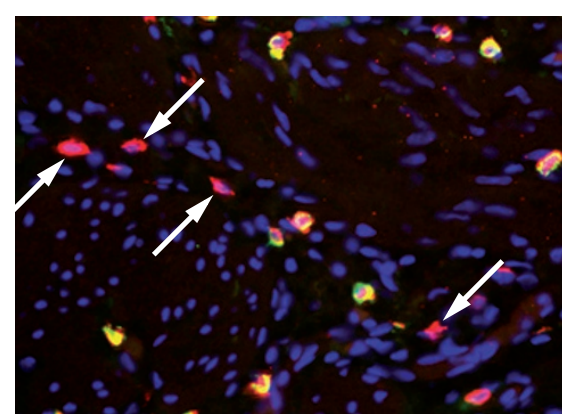

ICLCs (arrows) in the muscularis propria of the gallbladder. Image courtesy of M. Gajda.

that the results might have implications for future medical interventions for cholelithiasis - such as avoiding the use of drugs that might damage ICLCs (of which a few are known).

Isobel Franks

Original article Pasternak, A. et al. Loss of gallbladder interstitial Cajal-like cells in patients with cholelithiasis. Neurogastroenterol. Motil. doi:10.1111/nmo.12037 